ENCYCLOPEDEE Encyclopédie berbère

BERBERE

21 | 1999

21 | Gland - Hadjarien

\title{
Hadj Ahmed
}

(Elkhaj-Akhmed, El-Xag Axmed)

\section{P. Pandolfi}

\section{OpenEdition}

\section{Journals}

Édition électronique

URL : http://journals.openedition.org/encyclopedieberbere/1849

DOI : 10.4000/encyclopedieberbere.1849

ISSN : 2262-7197

\section{Éditeur}

Peeters Publishers

\section{Édition imprimée}

Date de publication : 1 septembre 1999

Pagination : 3289-3294

ISBN : 2-7449-0097-4

ISSN : $1015-7344$

\section{Référence électronique}

P. Pandolfi, «Hadj Ahmed », Encyclopédie berbère [En ligne], 21 | 1999, document H07, mis en ligne le

01 juin 2011, consulté le 24 septembre 2020. URL : http://journals.openedition.org/

encyclopedieberbere/1849; DOI : https://doi.org/10.4000/encyclopedieberbere.1849

Ce document a été généré automatiquement le 24 septembre 2020.

(c) Tous droits réservés 


\section{Hadj Ahmed}

(Elkhaj-Akhmed, El-Xag Axmed)

\section{P. Pandolfi}

1 El-Hadj Ahmed ag-el-Hadj-el-Bekri (Elkhaj Akhmed en tamâhaq) fut amenûkal de l'Ahaggar de 1861 à 1877. Il succéda à Ag-Mama ag Sidi et, à sa mort, fut remplacé à la tête des Kel-Ahaggar par Ahitayel ag Mokhammed-Biska, fils de sa tante maternelle.

\section{L'accession au pouvoir}

2 Le premier amenûkal connu des Kel-Ahaggar fut Salah à qui succéda son fils Mokhammed el-Kheir lui même remplacé, à la fin du XvIII ${ }^{e}$ siècle, par son fils Sidi. Ce dernier épousa Kella, considérée comme une descendante de Tin-Hinan et comme l'ancêtre fondatrice du groupe noble dominant (Kel yela) dans l'Ahaggar. Ce sont deux fils issus de cette union, Yunès et Ag-Mama ag Sidi qui se retrouvèrent ensuite à la tête des Kel Ahaggar. Il semblerait donc, comme l'avait justement relevé Benhazera (1908: 107), que les premiers dirigeants de l'Ahaggar se sont succédé selon un axe patrilinéaire. Ils étaient membres de « la plus importante tribu noble du Ahaggar » celle des Tégéhé-n-U-Sidi. Ces derniers, selon Duveyrier, appartenaient à la même lignée que les Imenân avec qui ils ne faisaient "qu'une même tribu... leur séparation n'indique qu'une bifurcation du même arbre.» (1864 p. 322). Quant à Yunès et Ag Mama, ils pouvaient se prévaloir tant de leur ascendance paternelle que de leur ascendance maternelle car fils de Sidi ag Mokhammed el-Kheir et de Kella.

3 Ag-Mama ag Sidi, le " doyen des centenaires du Sahara ", vivait encore en 1861 mais, aveugle et affaibli, il était dans l'incapacité de gouverner (Duveyrier 1864, p. 368). Aussi il devint nécessaire de lui trouver, de son vivant même, un successeur. Taytoq et Kelyela, les deux principaux groupes nobles de l'Ahaggar, vont alors s'affronter. Les premiers proposent Mokhammed, fils aîné d'Ag Mama et descendant de cette lignée d'où, depuis Salah, sont issus tous les imenûkalen (sg. amenûkal) de l'Ahaggar. Les Kelyela soutiennent eux Elkhaj-Akhmed qui deviendra effectivement le sixième amenûkal connu de l'Ahaggar. Le conflit Kel-үela/Taytoq qui se manifeste alors, y compris sous forme d'attaques armées réciproques, est ancien et remonte au moins au milieu du 
XVIII ${ }^{\mathrm{e}}$ siècle (voir A.O.M.22H73). Pour tenter de mettre fin à ces tensions, l'amenûkal Sidi ag Mokhammed el-Kheir avait déjà procédé à une nouvelle répartition des groupes tributaires entre les trois principales tribus nobles de l'Ahaggar et avait marié ses deux fils (Yunès et Ag-Mama) à des femmes Taytoq (Gast 1986). La lutte opposant ElkhajAkhmed et Mokhammed ag Ag-Mama ravive donc un conflit tenace. Après la nomination d'Elkhaj-Akhmed, de nombreux Taytoq et leurs tributaires (Tégéhé-n-Efis notamment) se réfugieront au Soudan où certains s'installeront de manière définitive. Surtout, depuis cette date, les Taytoq se retrouvent évincés de la compétition pour le pouvoir.

4 De par son ascendance maternelle, Elkhaj-Akhmed pouvait postuler au rang d'amenûkal. Sa mère (Zahra) était en effet l'aînée des six filles de Kella, la sœur de Yunès et Ag Mama. L'opposition entre Mokhammed ag Ag Mama et Elkhaj-Akhmed, la rivalité entre Taytoq et Kel-yela peuvent à ce niveau être interprétées comme un conflit entre deux modes de transmission. Pour accéder au pouvoir recherché, chacun de ces groupes a intérêt à mettre en avant soit une transmission du pouvoir en voie patrilinéaire soit une transmission en voie matrilinéaire. Or, il ne s'agit pas seulement de faire triompher le candidat présent mais aussi de s'assurer que la charge d'amenûkal n'échappera pas à son groupe dans le futur. En effet, si de par son ascendance paternelle, Mokhammed ag Ag Mama l'avait emporté, cela aurait également signifié que ses propres fils et ceux de son frère cadet, tous membres des Taytoq, auraient eu priorité à lui succéder. De même, le choix d'Elkhaj-Akhmed manifeste que la règle matrilinéaire l'emportant, l'amenûkal sera désormais choisi parmi les descendants masculins des filles de Kella et appartiendra (comme le démontre l'histoire des Kel-Ahaggar) aux Kel-үela.

Cependant, d'autres facteurs que sa place dans la descendance matrilinéaire de Kella furent nécessaires à Elkhaj-Akhmed pour assurer sa victoire. Les lignes consacrées par Duveyrier à la succession d'Ag Mama sont sur ce point éclairantes. Après avoir rappelé les difficultés auxquelles furent alors confrontés les Kel-Ahaggar, cet auteur précise qu'avec Elkhaj-Akhmed se trouvaient "miraculeusement réunies sur la tête d'un homme trois conditions importantes : le titre de marabout qui imposait le respect; la qualité d'étranger qui anéantissait toutes les rivalités locales ; la condition de fils d'une sœur de Guemâma. » (1864 p. 369).

6 Ainsi, outre l'ascendance maternelle, deux autres faits, d'ailleurs intimement liés, interviennent en faveur d'Elkhaj-Akhmed: "sa qualité d'étranger» et surtout son «titre de marabout ». Son père, El-Hadj-el-Bekri, appartenait en effet aux Ifoyas et les ascendants paternels d'Elkhaj-Akhmed se rattachaient, depuis plusieurs générations, à une lignée «maraboutique » bénéficiant d'un prestige politico-religieux très important dans l'ensemble du Sahara central. Son grand-père paternel, El-Hadj-el-Foqui, à qui de nombreux miracles sont attribués, était le fondateur de Timâsinin, site que développa par la suite le père d'Elkhaj-Akhmed. Ses oncles paternels, Sidi Moussa et Sidi Yemma, étaient également considérés comme des saints hommes, dépositaires de la baraka de la lignée et à l'origine eux aussi de nombreux miracles. Enfin, le frère d'Elkhaj-Akhmed n'était autre que le célèbre Cheikh Othman qui, à l'initiative de Duveyrier, entreprit un voyage en France durant l'année 1862 (Gardel 1961 et Pandolfi 1998).

7 Cette incontestable aura religieuse qui s'attachait à la famille d'Elkhaj-Akhmed semble avoir joué un rôle déterminant dans sa désignation comme amenûkal. Le poids important pris par la religion est d'ailleurs illustré par le fait que les religieux de Tombouctou sont intervenus en faveur d'Elkhaj-Akhmed (Duveyrier 1864 : 369). Ainsi, 
Sidi el-Bakkaï, marabout kunta dont l'influence s'étendait sur l'ensemble du Sahara, envoya un de ses frères dans l'Ahaggar même pour y soutenir Elkhaj-Akhmed.

Le choix d'Elkhaj-Akhmed marque tout à la fois la victoire des Kel-үela dans la lutte qui les opposait aux Taytoq et la reconnaissance de la primauté du principe de transmission matrilinéaire du pouvoir. Les Taytoq cependant n'accepteront jamais leur éviction du pouvoir et « tensions et jalousies n'ont cessé depuis lors d'empoisonner les relations entre Taytoq et Kel-yela. » (Gast 1986 p. 288).

Deux événements importants vont marquer le règne d'Elkhaj-Akhmed: la guerre fratricide qui opposera Kel-Ahaggar et Kel-Ajjer et la naissance des premiers centres agricoles de l'Ahaggar.

\section{La guerre contre les Kel-Ajjer}

10 Le conflit opposant Kel-Ahaggar et Kel-Ajjer trouve son origine dans la situation politique régnant dans l'Ajjer après l'éviction des Imenân par les Urayen. Ces derniers devenus les maîtres du pays persécutent les quelques Imenân continuant à y résider. Finalement, en 1872, ces derniers vont chercher refuge auprès des Kel-Ahaggar. Là, leurs femmes, dénommées timenukalin et fort réputées pour leur beauté, vont s'employer à réclamer vengeance auprès de leurs hôtes. «Les pleurs des belles timenukalin font leur effet sur les galants guerriers ahaggar ; la sultane Taber'out, par ses larmes, excite la compassion et le zèle des plus blasés, des moins vaillants. Ahitarel ag Mohammed Biska [...] déclare à la sultane : "Maintenant que tu es venue chez nous, je ne me reposerai pas sans avoir essuyé tes larmes." " (Gardel 1961 p. 148) De fait, en 1874, après l'échec d'une ultime tentative de conciliation, Elkhaj-Akhmed déclare la guerre aux Kel-Ajjer. Mais, il est plus que probable qu'il s'agisse là d'une cause conjoncturelle venue se greffer sur plusieurs rivalités de fond ayant pour enjeu le partage ou le monopole des bénéfices provenant du commerce caravanier. Opposition entre Kel-Ahaggar et Kel-Ajjer mais aussi, chez ces derniers, entre Urayen et Imanyasaten (cf. de Bary 1898). Ce conflit, «le fait le plus important de l'histoire de l'Ahaggar entre 1850 et 1900 " selon Foucauld (1925 p. 38), dura quatre ans. Ses péripéties furent nombreuses : victoire des Kel-Ahaggar sous les murs de Ghat en 1874, revanche des Kel-Ajjer unis à des arabes du Fezzan au combat de Tânhart où périrent de nombreux Imenân ainsi que yotman le fils d'Elkhaj-Akhmed (1875), combat d'Ugmîden qui vit la victoire des Kel-Ahaggar en 1877... On peut suivre le déroulement de cette guerre dans l'ouvrage du Lieutenant Gardel (1961) mais également par l'intermédiaire des nombreuses poésies composées, dans les deux camps, lors de ce conflit (voir Foucauld 1925 et 1930).

11 Très âgé et malade, Elkhaj-Akhmed, réfugié dans la Tàëssa, ne participa pas aux dernières expéditions et c'est sous la direction d'Ahitayel ag Mohammed-Biska, que les Kel-Ahaggar partirent au combat d'Ugmîden. Elkhaj-Akhmed mourut à la fin de l'année 1877 et fut enterré à Terhenânet. C'est Ahitayel ag Mohammed Biska (fils d'Amena troisième fille de Sidi et Kella) qui succéda à son cousin germain Elkhaj-Akhmed. Après le combat d'I-n-eleggi, cet amenûkal, aidé en cela par l'intervention de nombreux religieux qui servirent d'intermédiaires entre les différents camps en présence (de Bary 1898), conclura la paix avec les Kel-Ajjer. 


\section{L'initiateur de l'agriculture dans l'Ahaggar}

12 Jusqu'au milieu du XIX ${ }^{e}$ les terres de l'Ahaggar échappaient à l'agriculture et ce n'est qu'à partir des années 1840-1860, qu'eurent lieu les premières tentatives de mise en culture de terrasses alluviales (voir Gast 1968). À l'initiative de ces premiers essais agricoles, on trouve déjà celui qui deviendra quelques années plus tard amenûkal de l'Ahaggar : Elkhaj-Akhmed. C'est lui qui, vers 1820-22, suscita la première communauté agricole d'Idélès en incitant des esclaves à y travailler la terre. Mais, très vite, ces premiers essais avortèrent: les esclaves pressentis n'avaient ni l'expérience ni la «qualification technique » nécessaires à une telle entreprise (voir Barrère 1971). Les Kel-Ahaggar, toujours à l'initiative d'Elkhaj-Akhmed, allèrent chercher ailleurs ces «moniteurs agricoles » qui leur faisaient défaut. Ils firent appel à des agriculteurs du Touat et du Tidikelt et permirent à un certain nombre d'entre eux de venir se fixer sur leurs terres pour les cultiver. Ces nouveaux venus étaient des noirs sahariens dénommés harâtin (sg. hartâni) en langue arabe et izeggâyen (sg. azeggay) en tamâhaq. Se joignirent également à eux quelques Mrabtines (Ahl Azzi) communément appelés Kelyezzi dans l'Ahaggar. Grâce à leur maîtrise des techniques d'irrigation et notamment du système de drains par gravité (foggara en arabe, éfeli en tamâhaq), ils purent mettre en valeur certaines terrasses d'oueds et furent ainsi à l'origine des premières communautés rurales de l'Ahaggar. Rapidement, en effet, ces premiers venus demandèrent à leurs familles de les y rejoindre pour s'y fixer de manière définitive. Au début du siècle, le Père de Foucauld pouvait dénombrer environ 300 familles de cultivateurs « toutes originaires du Tidikelt et établies dans l'Ahaggar depuis moins de 50 ans » (1951 p. 632).

13 Cette venue et cette installation de gens originaires du Touat-Tidikelt peuvent s'expliquer par diverses raisons : vie misérable dans leur terroir d'origine, crainte de la puissance militaire des Kel-Ahaggar... Mais, il parait également indéniable que l'action d'Elkhaj-Akhmed qui pouvait jouer auprès de ses populations du prestige religieux reconnu à sa lignée fut ici déterminante. La tradition rapporte d'ailleurs qu'il lui suffit d'une takûté, offrande à Dieu d'un grand repas réunissant tous les habitants du ksar mrabtîn d'In-Salah, pour convaincre ceux-ci de déléguer un certain nombre des leurs en Ahaggar. Une seule condition restrictive fut posée : tabac et piment ne devaient pas y être cultivés afin de conserver à leur région d'origine le monopole de ces deux denrées.

Elkhaj-Akhmed favorisa également l'installation et le travail des cultivateurs notamment à Idélès et surtout Tazrouk où il pouvait compter sur la collaboration de son affranchi Karzika. C'est Elkhaj-Akhmed lui-même qui lors de l'ouverture de la première foggara en ce lieu fournit le magsur (une charge de mil, deux outres de beurre de chèvre, deux ou trois chèvres à égorger) destiné à nourrir l'équipe travaillant à ce projet. D'ailleurs, outre Karzika et quelques mrabtîn venus du Touat, tous les autres participants a cette première tentative agricole à Tazrouk étaient des esclaves appartenant à Elkhaj-Akhmed.

15 Cette politique novatrice, qui favorisa la venue et l'installation de personnes étrangères sur des terres qui leur avaient été jusqu'alors interdites, eut à terme des conséquences considérables sur l'histoire de l'Ahaggar. En effet, "le développement de ces communautés rurales a constitué une brèche de plus en plus large dans le système socio-politique et socio-économique des Touaregs. C'est à partir de l'existence des communautés rurales, devenues progressivement autonomes, que ceux-ci ont perdu la 
maîtrise des rapports de production et qu'un monde de sédentaires a peu à peu développé un autre type de civilisation technique et économique et une autre culture avec l'usage de la langue arabe. » (Gast 1987, p. 522).

\section{Le tombeau d'Elkhaj Akhmed.}

ElKhaj-Akhmed est enterré sur une petite butte située au milieu du lit de l'oued Terhenânet à environ $8 \mathrm{~km}$ en amont du village du même nom. Au sommet de cette butte, un muret de pierres sèches d'une hauteur de 60 à $80 \mathrm{~cm}$ délimite un cercle de 6 mètres de diamètre environ, qui entoure deux tombes. Elles sont toutes deux orientées vers le sud. La plus grande, à la tête de laquelle est planté un mât en bois de $190 \mathrm{~cm}$ de haut, est celle d'Elkhaj-Akhmed. Celle qui, de taille moins importante, se trouve à ses côtés est attribuée à son " secrétaire » (elkhodja) d'origine arabe. Il y a quelques années encore se trouvait près de ces tombes une grosse pierre ronde («comme un ballon ») qui servait aux ablutions sèches des pèlerins et avait la réputation de transmettre un peu de la baraka du défunt, tant il est vrai que la bénédiction divine ne disparaît pas après la mort des hommes qui la possèdent mais « subsiste dans la terre et les pierres le leurs tombeaux et dans tous les objets qui se rattachent au lieu funéraire. » (Nicolaisen $1961: 119)$

Dans le lit même de l'oued, entre la rive droite et la butte, a été délimitée à l'aide de pierres sèches une aire de prières (tamejjîda). Jusqu'au début des années 1970, les Dagyâli qui nomadisaient dans les environs avaient l'habitude de se réunir en cet endroit. Ces rassemblements ne suivaient pas un calendrier fixe. Ils étaient décidés, par le chef (ampar) et les principaux notables de la tribu, surtout quand une période de forte sécheresse se prolongeait. Comme nombre de marabouts du Sahara, Elkhaj-Akhmed est en effet réputé avoir une action bénéfique quant à la venue de la pluie tant désirée.

La plupart des participants arrivaient la veille au soir et établissaient leur campement au pied de deux gros rochers aux formes caractéristiques (semblables à de gros champignons) qui se trouvent à quelques 500 mètres en aval du tombeau sur la rive droite de l'oued. D'autres les y rejoignaient le lendemain matin. Quand tout le monde était réuni, on égorgeait plusieurs chèvres en l'honneur de l'amenûkal défunt. Puis les hommes participaient à une prière collective dans l'aire prévue à cet effet; les femmes $\mathrm{y}$ assistaient, elles, à distance. Un des « anciens » enduisait de beurre de chèvre le mât en bois planté à la tête de la tombe. Ce cérémonial terminé, un repas (takûté) au cours duquel étaient consommées les chèvres sacrifiées, réunissait les personnes présentes sur le lieu du bivouac. Quelque temps après, tous les participants rejoignaient leurs campements respectifs parfois très éloignés de cet endroit.

Suite à la sédentarisation des Dag-үâli dans des centres éloignés les uns des autres et surtout des pressions et mises en demeure - au nom de l'islam - venues de l'extérieur, ces pèlerinages ont cessé à partir de 1973-1974. Mais depuis 1993 un rassemblement sous forme de ziara est à nouveau organisé auprès du tombeau d'Elkhaj-Akhmed à l'initiative de ses descendants. Quoi qu'il en soit ce tombeau est toujours demeuré pour nombre de Kel-Ahaggar un lieu privilégié de dévotion. Haltes et prières y sont fréquentes pour les gens passant à proximité. Souvent on en profite pour formuler un vœu ou faire appel à l'action bénéfique du défunt. Outre la venue de la pluie, la baraka de ce dernier est en effet jugée très efficace en cas de disparition d'un objet ou d'un animal. Nombreux sont les récits qui illustrent un tel pouvoir (Pandolfi 1998). Elkhaj- 
Akhmed y apparaît davantage comme un personnage hors du commun, comme un homme bénéficiant de pouvoirs surnaturels que comme un amenûkal parmi d'autres. En ce sens, il prend place dans la mémoire Kel-Ahaggar aux côtés de Mûsa ag Amastan (Gast 1961), lui aussi considéré comme un être qui aurait transcendé la simple condition humaine.

\section{BIBLIOGRAPHIE}

ARCHIVES D'OUTRE-MER, Aix en Provence, Carton $22 \mathrm{H} 73$.

BARRERE G, Problèmes économiques d'un centre de culture d'altitude au Sahara : Idélès, Journal d'agriculture tropicale et de botanique appliquée, XVIII, 12, 1971, p. 540-565.

BARY E., von. Le dernier rapport d'un européen sur Ghât et les Touareg de l'Aïr (traduit et annoté par H. Schirmer), Paris, Librairie Fischbacher, 1898, 222 p.

BENHAZERA M., Six mois chez les Touaregs du Ahaggar, Alger, A. Jourdan, 1908, 233 p.

DUVEYRIER H., Les Touareg du Nord, Paris, Challamel, 1864, 488 p.

FOUCAULD Ch. de, (recueillies par), Poésies touarègues. Dialecte de l'Ahaggar, Paris, Leroux, 1925,1.1, 658 p., t. II, 1930, 461 p.

FOUCAULD Ch. de., Dictionnaire touareg-français, dialecte de l'Ahaggar, Paris, Imprimerie Nationale, 4 vol. , 1951-52, $2028 \mathrm{p}$.

GARDEL G., Les Touareg Ajjer, Alger, Baconnier, 1961, 388 p.

GAST M., « Histoire de Moussa ag Amastane par Khabti ag Abahag », Bulletin de Liaison Saharienne, 41,1961 , p. 71-77.

GAST M., Alimentation des populations de l'Ahaggar, Paris, A.M.G., 1968, 458 p.

GAST M., « Histoire des Kel-Ahaggar », Encyclopédie berbère, III, 1986, p. 282-303.

GAST M., « Alimentation au Sahara central », Encyclopédie berbère, IV, 1987, p. 515-528.

NICOLAISEN J., « Essai sur la religion et la magie touarègues », Folk, 3, 1961, p. 112-162.

PANDOLFI P., Les Touaregs de l'Ahaggar, Paris, Karthala, 1998, 473 p.

INDEX

Mots-clés : Touareg 